\title{
Memory for word location during reading: Eye movements to previously read words are spatially selective but not precise
}

\author{
ALBRECHT W. INHOFF and ULRICH W. WEGER \\ State University of New York, Binghamton, New York
}

\begin{abstract}
In two experiments, readers' use of spatial memory was examined by asking them to determine whether an individually shown probe word had appeared in a previously read sentence (Experiment 1) or had occupied a right or left sentence location (Experiment 2). Under these conditions, eye movements during the classification task were generally directed toward the right, irrespective of the location of the relevant target in the previously read sentence. In two additional experiments, readers' knowledge of prior sentence content was examined either without (Experiment 3) or with (Experiment 4) an explicit instruction to move the eyes to a target word in that sentence. Although regressions into the prior sentence were generally directed toward the target, they rarely reached it. In the absence of accurate spatial memories, readers reached previously read target words in two distinct steps-one that moved the eyes in the general vicinity of the target, and one that homed in on it.
\end{abstract}

Fluent reading requires the mastery of task-specific skills, since the written language signal differs fundamentally from the spoken language signal. To-be-read material often consists of a multitude of concurrently available linguistic symbols, and readers must learn to select in a principled manner from among them so that sentence meaning can be determined. Since high-acuity vision is confined to a relatively small retinal area encompassing one to two words, this selection process requires the execution of a sequence of eye movements (saccades). Most saccades follow word order, but exceptions occur, and some saccades (regressions) move the eyes in a direction that is opposite to word order.

According to Rayner and Pollatsek (1989), from 15\% to more than $25 \%$ of all eye movements during reading are regressions. Approximately one quarter of them move the eyes to a different location within a word. The remaining regressions move the eyes to a prior word, with the vast majority of them being directed at the word immediately to the left of fixation (Hogaboam, 1983; Vitu $\&$ McConkie, 2000). Occasionally, readers also execute a relatively large regression that moves the eyes across a considerable distance to an earlier segment of the text (Kennedy \& Murray, 1987).

Corpus analyses indicate that the programming of regressions differs from the programming of forwarddirected saccades. Forward-directed saccades can be pre-

This work was supported by NIH Grant R01HD043405. We thank Robert Medl, now with IBM, for the collection of the data in Experiments 1 and 2. We also thank Ralph Radach, Alan Kennedy, and Alexander Pollatsek for helpful comments on an earlier version of the manuscript. Correspondence concerning this article should be sent to A. W. Inhoff, Department of Psychology, State University of New York, Binghamton, NY 13902 (e-mail: inhoff@binghamton.edu). ceded by short-duration fixations, and they show characteristic effects of landing position (Rayner, 1979) and launch site (McConkie, Kerr, Reddix, \& Zola, 1988). These saccades typically position the eyes between the beginning and the center of a to-be-read word, its preferred landing position (Rayner, 1979). The landing position is adjusted to the right of a word's center when the launch site was relatively close and to the left of the center when the launch site was relatively far (McConkie et al., 1988). Regressions of up to 10 character spaces are rarely preceded by short-duration fixations, by contrast, and the eyes' mean landing position on a word following a regression is close to the word center for launch distances of up to 10 letter spaces (Radach \& McConkie, 1998). The programming of a regression thus appears to be functionally distinct from the planning of a forwarddirected saccade.

Strikingly, interword regressions do not generally prevent the global process of meaning extraction. Kolers (1968) noted that "the information that is picked up in a regressive movement is not allowed to distort the syntax of the sentence being read; rather, it supplements or fills out what has already been read. ... Otherwise, the reader would be dealing with a word-salad, an incomprehensible sequence of syntactically anomalous words, rather than a coherent message" (pp. xviii-xix). According to Kolers, readers must "keep in mind" what has been read up to the point at which a regressive saccade is executed. The programming of a regression thus appears to differ from the programming of a forward-directed saccade, in that readers use a stored representation of previously read text to guide the eyes.

Zechmeister, McKillip, Pasko, and Bespalec (1975) and, later, Kennedy (1987, 1992, 2000; Kennedy, Brooks, Flynn, \& Prophet, 2003; Kennedy \& Murray, 1987; see 
also Fischer, 2000) proposed that the representations of linguistic units are spatially indexed and that these spatial indexes are used to program spatially selective regressions. According to Kennedy and Murray's (1987; Kennedy, 1992) spatial-indexing hypothesis, readers assign spatiotopic values either to linguistic tokens (letter strings, words, or phrases) or to points in space where certain cognitive operations take place. Spatiotopic values become an integral part of a text representation, and they make linguistic knowledge spatially addressable. According to this view, the spatial index of a previously read word is used to direct the eyes to it, which may be advantageous when comprehension difficulties can be traced to that word during the reading of subsequent text. The spatial-coding hypothesis assumes that the page layout or screen display functions as an external memory (Kennedy et al., 2003) that helps to establish spatial coordinates.

Knowledge of the precise spatial location of a word could prevent "word-salad" after a regression, because the temporal order of the word input can be mapped into a location-based representation of the text: "It is this spatial knowledge that allows for the recovery of a temporally coded event. That is, only by knowing where words lie can the reader be completely freed from the requirements to inspect them in a strictly controlled order" (Kennedy, 1992, p. 382). For instance, a fixation of word $N$ may be followed by a regressive eye movement to the prior word $N-1$, either because this word was not fully identified (Vitu \& McConkie, 2000) or because its rereading may assist in the resolution of processing difficulties during the reading of word $N$. In case of an interword regression, spatiotopic indexes can be used by the syntactic processor to determine that word $N-1$ does not follow word $N$ in the to-be-constructed sentence representation. Furthermore, after a regression, spatiotopic indexes can direct the eyes to the next to-be-processed word in the text, such as word $N+1$ when the fixation of word $N-1$ has been completed.

Empirical support for the spatial-indexing hypothesis has taken several forms. After reading a passage, readers can, on occasion, recall where on a page specific linguistic information was conveyed even when the linguistic information itself cannot be retrieved (Zechmeister \& McKillip, 1972; Zechmeister et al., 1975). Other measures provide converging evidence. Readers tend to move the eyes to a previously read sentence region that is critical for the disambiguation of a subsequently encountered ambiguous phrase (Carpenter \& Daneman, 1981; Ehrlich \& Rayner, 1983; Frazier \& Rayner, 1982; Rayner \& Frazier, 1987), and they reinspected a previously read passage segment to answer passage-related questions (Christie \& Just, 1976). Kennedy's experiments (Kennedy et al., 2003; Kennedy \& Murray, 1987) have provided particularly striking evidence for the spatial indexing of previously read words during reading. In one influential experiment (Kennedy \& Murray, 1987), readers were asked to read a sentence of text that occu- pied a single line. Three seconds after sentence onset, a word appeared to the right of the last word of the sentence, and the readers were asked to determine whether this word had appeared in the previously read sentence. The word shown to the right was used in the previously read sentence on positive trials; a synonymous word was used in the sentence on negative trials. Under these conditions, the readers executed long-range regression of up to 60 character spaces that moved the eyes onto the corresponding word in the sentence or its synonym. The incidence of these long-range regressions was relatively low, occurring on only $6 \%$ of the trials. A considerably larger proportion of highly accurate long-range regressions, ranging from slightly more than $10 \%$ to approximately $50 \%$, occurred in Kennedy et al. . Even more general support for the spatial-indexing hypothesis was provided by Baccino and Pynte (1994), who examined readers' ability to backtrack an anaphoric referent during passage reading. Instead of recording eye movements, readers were asked to manually point at the antecedent, which they did with a remarkable degree of accuracy.

Precisely how readers record and store the spatial location of previously read words is not well specified, however. Considering the evidence in support of spatial indexing, Kennedy et al. (2003) noted that "even if it is concluded that some form of spatial coding is implicated in successful reading, we are largely ignorant as to its nature, its coordinate structure, its duration and its precise function" (p. 194). Moreover, there are data that suggest that spatial indexing may not occur during normal reading and that its occurrence may depend on relatively unique experimental conditions.

In Therriault and Raney's (2002) study of passage reading, memory for the location of information on a page was lower than memory for the sequence of information in the text, and memory for location did not improve when readers were explicitly instructed that it would be tested. In Christie and Just's (1976) study, requests for spatial information were answered more slowly than requests for linguistic information. As they pointed out, substantially longer latencies for the retrieval of location-specific information suggested that it was less accessible than content-specific linguistic information. Consistent with this, Rawson and Miyake (2002) found that readers' linguistic abilities, but not their visuospatial abilities, were correlated with word localization performance.

Thus, the spatial-indexing hypothesis may need to be put on its head, in that it may be linguistic knowledge, rather than spatial knowledge, that is used to infer the location of words that are relatively distant from the current fixation. Readers may have some short-lived memory for the precise spatial location of a previously read target word, so that regressions can be directed to a precise location if it is up to 10 character spaces to the left of fixation (Radach \& McKonkie, 1998). As the eyes move farther away from a regression target, verbal memory may be used to reconstruct the location of a previously read word. A reader who can determine that such 
a word occurred near the beginning of a previously read single-line sentence can use this knowledge to direct the eyes toward the left-side segment of the line of text. Conversely, knowledge that the target word appeared near the end of the sentence can be used to direct the eyes to its right-side segment. Such an account can explain the lower accuracy (Therriault \& Raney, 2002) and the longer response latency for spatial than for content-based queries (Christie \& Just, 1976). It can also explain the robust correlation between verbal memory and spatial localization performance (Rawson \& Miyake, 2002). It cannot explain, however, the remarkable spatial accuracy of longrange regression in Kennedy's (Kennedy et al., 2003; Kennedy \& Murray, 1987) experiments.

The main goal of the present study was to determine whether recognition of a word in reading goes along with its spatial indexing and whether readers can use the spatial index of a previously read word to reach it with a single accurate regression. Experiments 1 and 2 were performed to examine whether the eyes are moved to the location of a previously recognized target word when it becomes relevant in a subsequent decision task. Experiments 3 and 4 were performed to examine whether regressions are accurately directed at a previously read target word when it becomes relevant during subsequent sentence reading.

\section{EXPERIMENT 1}

One of the implications of the spatial-indexing hypothesis is that knowledge of the spatial location of a linguistic unit is an integral component of its representation in working memory. Positioning the eyes at the spatial location of a previously read word should thus assist retrieval of other information. As a result, readers should generally seek to revisit the spatial location of a target word when they try to retrieve information about it, as has occurred in studies in which the retrieval of previously shown nonlinguistic items has been examined (Hall, 1974; Richardson \& Spivey, 2000).

To determine whether readers direct the eyes toward the location of a previously read target when it becomes relevant at a later point in time, participants read individual sentences, each of which contained a target word that occupied a location either near the sentence beginning (i.e., near the left side of the screen) or near the sentence ending (i.e., near the right side of the screen). Sentence reading was followed by a recognition task in which the participants were asked to determine whether a single (probe) word, shown near the center of the screen, matched a word (the target) in the previously read sentence. The probe and the target were either identical or close semantic associates. If probe recognition involved retrieval of the previously read target's spatial index under these conditions, readers should direct the eyes toward the prior target location, since its fixation may assist in the retrieval of useful linguistic information.

\section{Method}

Participants. Twelve undergraduate students at the State University of New York at Binghamton either were paid or received experimental course credit for their participation. All the participants had normal vision and were naive as to the purpose of the experiment.

Apparatus. The participants were tested in a sound-insulated, dimly illuminated room. A $60-\mathrm{Hz}$ noninterlaced VGA monitor was used to display text in VGA $(640 \times 480$ pixels $)$ mode. Courier font was used, with text being shown in light green on a black background. The distance between the readers' eyes and the monitor was set at $70 \mathrm{~cm}$; at this viewing distance, each letter of text subtended approximately $0.33^{\circ}$ of visual angle.

Eye movements were recorded via a fifth-generation dual-Purkinje eye-tracking system. Viewing was binocular, but eye movements were recorded from the right eye only. The system had a relative visual resolution of $10 \mathrm{~min}$ of arc, and its output was linear over the vertical and horizontal range of the visual display. The computer controlled the visual display and stored horizontal and vertical fixation coordinates every $2 \mathrm{msec}$. A Logitech mouse and QWERTY keyboard with a numeric keypad were also interfaced with the computer. The mouse was used to control the onset and offset of visual displays, and the keyboard was used to monitor the readers' performance in the word recognition task, since they were instructed to press the number 1 key for yes/same responses and the number 3 key for no/different responses.

Procedure. The experiment began with a calibration of the eyetracking system for each participant. During calibration, the participant was requested to fixate four monitor positions (left top, right top, left bottom, and right bottom) as they were sequentially illuminated on the screen. A bite bar was used by each participant while eye movements were recorded, to reduce head movements. The reader was asked to fixate each illuminated position and to depress a mouse key when the position was accurately fixated. After each keypress, the computer calculated the mean fixation location during the $150 \mathrm{msec}$ immediately following mouse activation. After calibration was completed, six character-size fields were shown (top-left, top-right, bottom-left, and bottom-right, 2 in. to the left of the screen center and 2 in. to the right of the screen center), and the reader's fixation location was shown on the screen as a bright onecharacter cross that moved in synchrony with the eyes. The reader was asked to fixate all six spatial positions, irrespective of the position of the eye-movement-contingent white cross. The calibration was considered successful when the computer-generated eye position (the white cross) deviated by no more than one character space from the actual eye position (the illuminated character space). Recalibrations were performed when larger errors were observed.

After successful calibration, the participant was asked to fixate a one-character-size marker at the left side of the screen and to use the left hand to depress a mouse button to display a one-line sentence. Each sentence contained a target word, which occurred near either the left or the right side of the screen. Reading for sentence meaning was encouraged, and the participants were asked on approximately every 10th sentence to repeat or paraphrase the most recently read sentence. Completion of sentence reading was followed by another mouse press that terminated the visibility of the sentence. A third mouse press displayed a fixation marker, consisting of a sequence of Xs, at the horizontal screen center. The participant was asked to fixate the string and to press the mouse button once more. This replaced the letter string with a length-matched source word that matched the target (on yes/same trials) or a lengthmatched semantically associated word that did not match the target (on no/different trials). Eye movements were monitored during sentence reading and during the subsequent probe decision task. The participants had to decide whether the probe was old or new and performed this classification task with the right hand, which rested on the numeric keypad of the keyboard outside the participants' 
range of view. After the choice was completed, the participants pressed a mouse button with the left hand to initiate another trial.

Materials and Design. Twenty-eight one-line declarative 9- to 13-word sentences were constructed. Each of the sentences contained a target location either near the sentence beginning (and therefore, near the left side of the screen), or near the sentence ending (and thus, near the right side of the screen). The target location could be occupied by one of two closely related target words. Both words of a target pair were matched on length and familiarity, both were semantically associated, and both fit into sentence context. Two sentence versions were created that were identical except that the target word in one version was replaced with a semantically associated alternative target in the second version. Each sentence was followed by the presentation of a fixation marker and a probe word that was either identical to the target word or semantically associated.

Four lists were created, each containing a different version of the four possible target by probe word combinations. On each list, half of the sentences contained a right-side target, and the remaining sentences contained a left-side target. Orthogonal to this, half of the sentences were followed by a same probe word, and half were followed by a different probe word. An example of the four versions of a right-side probe word is shown in Figure 1.

Measurements. Fixation durations of less than $50 \mathrm{msec}$ and more than $1,000 \mathrm{msec}$ were excluded, resulting in the removal of less than $1 \%$ of the data. Trials were also excluded when a track loss occurred prior to or during target reading, which occurred on approximately $5 \%$ of the trials. Viewing durations on targets on eligible trials were measured while the sentence was read. A target was considered fixated when the line of sight fell on one of its constituent letters or the blank space preceding it. Two standard measures of visual word recognition during sentence reading - skipping rate and gaze duration (Inhoff \& Radach, 1998; Inhoff \& Weger, 2003; Rayner, 1998) - were evaluated in order to index the success with which target words were identified during sentence reading. Skipping rates consisted of the relative frequency with which the subject's eyes jumped over (skipped) a target word during sentence reading, and gaze durations consisted of the time spent fixating on a target word until the participant fixated on another word. Four additional measures were obtained to index performance in the samel different classification task. They consisted of the direction and latency of the lateral saccade that moved the eyes to the right or left off the probe word and the accuracy and latency of the manual classification performance.

\section{Results}

Sentence reading. Target skipping rates were equivalent for the two sentence locations, occurring during the reading of $21 \%$ and $18 \%$ of left- and right-side targets, respectively $[t(11)<.5$.] Similarly, gaze durations for left-side targets were virtually identical to gaze durations for right-side targets [435 vs. $434 \mathrm{msec}$, respectively; $t(11)=0]$. These results show that there were no intrinsic differences between the reading of a target word when it occupied a right- or a left-side screen location.

Decision task. An inspection of eye movements during probe word viewing showed that readers never directed their eyes to the precise location of the previously read target or its semantic associate. Yet their eyes were not completely stable. On approximately $5 \%$ of the trials, the readers executed a small lateral saccade to the right or left off the probe word, with a mean size of approximately three character spaces. An analysis of variance (ANOVA) with target location and probe-target rela-

Version I: Sample sentence with a right-side target:

I received a telephone call from my father on Wednesday.

Center Fixation:

\#\#\#\#\# (fixation)

Probe Word:

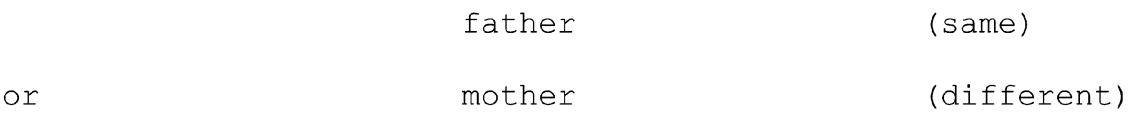

Version II: Sample sentence:

I received a telephone call from my mother on Wednesday.

Center Fixation:

\#\#\#\#\# (fixation)

Probe Word:

$\begin{array}{ll}\text { mother } & \text { (same) } \\ \text { or father } & \text { (different) }\end{array}$

Figure 1. The sequence of events during an experimental trial in Experiment 1. 
tionship of factors showed that the frequency of saccades of the probe was independent of the location of the previously read target word and of the probe-target relationship (both $F \mathrm{~s}<1$; see Table 1).

An additional ANOVA in which the direction of the saccade to the right or left off the probe was considered showed that the vast majority of these saccades were directed toward the right $[F(1,11)=26.97, p<01]$, irrespective of the spatial location of the previously read target. Specifically, a saccade to the right occurred on $11 \%$ of the trials in which the target had been to the left of the probe and on $8 \%$ of the trials in which it had been to its right. Saccades leaving the probe for a left-side location were equally rare for previously encountered right- and left-side targets ( $1 \%$ each). The two-way interaction of target location and saccade direction and the three-way interaction of target location, saccade direction, and probe type (same vs. different) were negligible (both $F<1)$.

Saccade latencies were extremely variable within and across readers, ranging from $174 \mathrm{msec}$ to more than $5,000 \mathrm{msec}$, with a grand mean and grand standard deviation of 1,050 msec and $494 \mathrm{msec}$, respectively. Due to the relatively small number of saccades, interpretable mean latency values could not be computed.

Manual decision accuracies and decision latencies, as a function of target location and probe type (same vs. different), are also shown in Table 1. Classification was quite accurate, exceeding $90 \%$ in all conditions. A statistical analysis of accuracy rates as a function of prior target location and probe type (same vs. different) revealed no reliable effect (all $\left.F_{\mathrm{s}}<1\right)$. The corresponding analysis of manual classification latencies revealed shorter reaction times for same than for different trials $\left[F(1,10)^{1}=\right.$ $14.09, p<.001]$ but no other reliable effect (all $F_{\mathrm{S}}<1$ ).

\section{Discussion}

The main results of Experiment 1 showed that lateral saccades during a classification task were not spatially selective. Saccades leaving the probe during the decision task were generally directed to the right, irrespective of the location of the target in the previously read sentence. Moreover, those saccades that moved the eyes in the direction of a previously visible target word were too small to reach its prior location.
Although these findings are not in harmony with the spatial-indexing hypothesis, it could be argued that the frequency with which saccades moved off the probe was too low to be sensitive to target position effects. Moreover, the demands of the decision task may have undermined the use of spatial memory in Experiment 1. Words may have been spatially indexed during sentence reading, but these indexes may not have been used, either because the task did not require any use of spatial knowledge or because spatial indexing is process specific. Specifically, spatial indexes may be applied to linguistic tokens only when processing difficulties arise (Kennedy \& Murray, 1987). In Experiment 2, these two alternatives were examined.

\section{EXPERIMENT 2}

Experiment 2 was similar to Experiment 1 in that it sought to determine whether the retrieval of a previously read word after sentence reading activated the word's spatial index. It differed from Experiment 1 in that an experimental task was used that explicitly required the use of spatial knowledge and in that it sought to determine whether the spatial indexing of words is process specific. As in Experiment 1, sentence reading was followed by a decision task in which a centrally presented probe was to be related to a previously read target word that had occupied either a right- or a left-side sentence location. In contrast to Experiment 1, each to-be-classified probe word had been a constituent of the previously read sentence, and the readers were asked whether it had occupied a left- or a right-side sentence location. Second, different types of target words were used, consisting of low-frequency words that should be difficult to recognize, high-frequency words that should be relatively easy to recognize, and functor words that should be particularly easy to encode (Greenberg \& Koriat, 1991; Healy, 1994). If spatial indexing is a function of task demands, spatially selective saccades may occur when the classification task requires spatial evaluation. Moreover, if spatial indexing is a function of the ease of target identification and encoding, spatially selective saccades should occur most often when low-frequency targets are classified and least often when functor words are classified. The latter prediction is based on the claim that low-frequency

Table 1

Experiment 1: Same-Different Decision Latencies (in Milliseconds), Decision Accuracies (in Percentages), and Relative Frequencies (in Percentages) With Which Lateral Saccades Occurred Prior to the Same-Different Decision (With Standard Errors)

\begin{tabular}{|c|c|c|c|c|c|c|c|}
\hline \multirow{2}{*}{$\begin{array}{l}\text { Target Word Location } \\
\text { During Reading }\end{array}$} & \multirow{2}{*}{$\begin{array}{l}\text { Probe-Target } \\
\text { Relationship }\end{array}$} & \multicolumn{2}{|c|}{ Probe RTs } & \multicolumn{2}{|c|}{$\begin{array}{c}\text { Probe } \\
\text { Accuracy }\end{array}$} & \multicolumn{2}{|c|}{$\begin{array}{l}\text { Frequency } \\
\text { of Saccades }\end{array}$} \\
\hline & & $M$ & $S E$ & $M$ & $S E$ & $M$ & $S E$ \\
\hline \multirow[t]{2}{*}{ Left side } & Same & 1,434 & 72 & 95 & 0.01 & 4.1 & 7 \\
\hline & Different & 1,856 & 122 & 92 & 0.02 & 6.4 & 15 \\
\hline \multirow[t]{2}{*}{ Right side } & Same & 1,507 & 148 & 94 & 0.02 & 4.3 & 3 \\
\hline & Different & 1,757 & 151 & 91 & 0.02 & 3.7 & 4 \\
\hline
\end{tabular}


words attract more attention (e.g., Malmberg \& Nelson, 2003 ) and the finding that low-frequency words require longer viewing durations (e.g., Inhoff \& Rayner, 1986).

\section{Method}

Participants. Twelve undergraduate students at the State University of New York at Binghamton participated for pay or experimental course credit. None of the subjects had participated in Experiment 1 .

Apparatus, Procedure, Measures, and Analyses. The apparatus, procedure, measures, and analyses in Experiments 1 and 2 were largely identical, except for small changes in the materials and the experimental task. The materials now contained different target types, and the readers were asked to determine whether a probe word had occupied a left- or a right-side target location during prior sentence reading.

Materials and Design. Seventy-two one-line sentences, each containing 9-13 words, were used. Each sentence contained two possible targets, one positioned to the left side of the horizontal screen center and the other positioned to its right. Forty-eight of the target sentences contained 2 content (open-class) words, 1 with a frequency of occurrence of 30 or less per million (Kučera \& Francis, 1967) and 1 with a frequency of more than 30 per million (referred to as low- and high-frequency words, respectively). Half of the low- and high-frequency target words appeared near the left side of the screen, and the remaining targets appeared near its right. Twenty-four sentences contained two functor (closed-class) targets, one appearing to the left of the screen center and one appearing to its right. Figure 2 shows sentences with content and functor word targets, as well as the subsequently presented source word.
Two probe word versions were constructed for each sentence, one in which the probe corresponded to the left-side target, and one in which it corresponded to the right-side target. Two lists were constructed, each containing a different sentence-probe pairing. Half of the probes on a list referred to a right-side target, and the other half referred to a left-side target. On each list, the ordering of right- and left-side reference locations and of word types was randomized.

\section{Results}

Sentence reading. Target skipping rates and gaze durations are shown in Table 2 as a function of target type and sentence location.

Skipping rates were a function of target type $[F(2,22)=$ $29.16, p<.001]$. Paired comparisons revealed substantially more skipping for functor words than for the two types of target words (both $p \mathrm{~s}<.01$ ); the difference between high- and low-frequency targets was not reliable $[F(1,11)=2.22, p>.16]$. As in Experiment 1, the main effect of sentence location was negligible, and sentence location did not interact with target type (both $F<1$ ).

Gaze durations on target words also showed an expected effect of processing difficulty $[F(2,22)=38.54$, $p<.001]$. Paired comparisons revealed significant differences between all three word types, with the shortest gaze durations on closed-class functor words and the longest gaze durations on low-frequency content words

Sample sentence with a content target:

I'll take the lone puppy from the five boys who were teasing it.

Center Fixation:

$$
\text { \#\#\#\# }
$$

Probe Word:

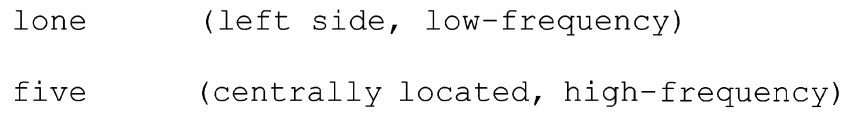

Sample sentence with a functor target:

I want the peas and the potatoes but I can't eat them both.

Center Fixation:

Probe Word:

$\begin{array}{ll}\text { and } & \text { (left side) } \\ \text { but } & \text { (centrally located) }\end{array}$

Figure 2. The sequence of events during an experimental trial in Experiment 2. 
Table 2

Experiment 2: Skipping Rates, Gaze Durations on Target Words During Sentence Reading, and Performance in the Decision Task, Consisting of the Relative Frequency of Lateral Saccades Decision Accuracy, and Decision Latency as a Function of Word Type and Target Location (With Standard Errors)

\begin{tabular}{|c|c|c|c|c|c|c|c|c|c|}
\hline \multirow[b]{2}{*}{ Target Type } & \multirow{2}{*}{$\begin{array}{l}\text { Sentence } \\
\text { Location }\end{array}$} & \multicolumn{2}{|c|}{ Skipping } & \multicolumn{2}{|c|}{ Gaze Duration } & \multicolumn{2}{|c|}{ Decision Accuracy } & \multicolumn{2}{|c|}{ Decision Time } \\
\hline & & $M$ & $S E$ & $M$ & $S E$ & $M$ & $S E$ & $M$ & $S E$ \\
\hline \multirow[t]{2}{*}{ Low frequency } & Left & .20 & .06 & 390 & 38 & 99 & 0.01 & 1,290 & 131 \\
\hline & Right & .21 & .06 & 403 & 19 & 89 & 0.03 & 1,694 & 294 \\
\hline \multirow[t]{2}{*}{ High frequency } & Left & .19 & .05 & 336 & 28 & 97 & 0.02 & 1,354 & 152 \\
\hline & Right & .15 & .04 & 366 & 14 & 92 & 0.02 & 1,552 & 167 \\
\hline \multirow[t]{2}{*}{ Functor word } & Left & .47 & .07 & 279 & 17 & 95 & 0.02 & 1,774 & 277 \\
\hline & Right & .49 & .04 & 263 & 12 & 90 & 0.03 & 1,930 & 319 \\
\hline
\end{tabular}

(all $p<.025)$. Importantly, the effect of the target word's sentence location (left vs. right) was quite small and not reliable $(F<1)$, as was the interaction of target type and sentence location $[F(2,22)=1.51, p>.24]$. The sentencereading data thus revealed substantial differences in the ease of target recognition but no intrinsic differences between right and left sentence locations during sentence reading.

Decision task. As in Experiment 1, the readers did not move their eyes to the previously visible target. Instead, small lateral movements occurred, with a mean saccade size of less than three character spaces. As can be seen in Table 3, task type had a profound effect on lateral saccades, which were much more common than they were in Experiment 1, occurring on more than one fifth of the trials.

A 3 (word type) $\times 2$ (target location) $\times 2$ (saccade direction) ANOVA revealed a robust effect of saccade direction. As in Experiment 1, saccades to the right off the probe were much more common than saccades to the left $\left[F(1,10)^{2}=19.36, p<.01\right]$. Word type influenced frequency of saccades, which were more common for functor words than for either high- or low-frequency content words $[F(2,20)=17.64, p<.01]$. Saccade direction and word type also interacted, with saccades to the right being particularly common for functor word $[F(2,20)=$ $5.37, p<.025]$. Critically, the interaction of target location and saccade direction was negligible $(F<1)$. Saccades to the right were equally common for left- (34\%) and right-side (34\%) targets. Although much less common, saccades to the left of the probe also occurred with equal frequency for left- and right-side targets (4\% and $3 \%$, respectively). This effect pattern occurred irrespective of word type, and the three-way interaction of saccade direction, target location, and word type was negligible $(F<1)$.

Decision accuracy and manual decision latencies are also shown in Table 2 . Both measures revealed effects of prior target location, with more accurate and faster responses when the target occupied a left- rather than a right-side sentence location $\left[F(1,8)^{2}=8.08\right.$ and $F(1,11)=$ 6.63 , respectively; both $p \mathrm{~s}<.025]$. Decision latencies were also a function of word type being longer for functor words than for content words. Subanalyses revealed significant differences between functor words and the two types of content words (both $p<.01$ ), but not between high- and low-frequency content words $(p>.15)$.

\section{Discussion}

The sentence reading data in Experiment 2 showed familiar effects of word frequency, with relatively long gaze durations for low-frequency words and the shortest gaze durations for functor words. Word type also influenced decision latencies and the frequency of saccades off the probe word, decision latencies being relatively long and movements off the target being relatively common for functor words, since the longer decision latencies for functors provided more opportunity for the execution of saccades during probe viewing. Critically, the direction of saccades leaving the probe during the decision task was not influenced by the target's prior location in the sentence, and this was independent of the ease with which a target word was processed during sentence reading. The results of Experiment 2 are thus not in harmony with either a general or a process-specific account of the spatial-indexing hypothesis.

A comparison of saccade frequencies in Experiments 1 and 2 showed that movements off the probe word occurred significantly more often in Experiment $2[F(1,21)=$ $26.01, p<.01]$, indicating that the lack of support for the spatial-indexing hypothesis in this experiment cannot be attributed to the paucity of lateral saccades to the right or left of the probe during the decision task. Moreover, the

Table 3

Experiment 2: Relative Frequency of Saccades (in Percentages) in the Spatial Classification Task as a Function of Word Type, the Word's Prior Sentence Location, and the Direction of the Saccade When a Saccade Was Executed While the Task Was Executed (With Standard Errors of the Means)

\begin{tabular}{cccccccc}
\hline & Sentence & & \multicolumn{2}{c}{$\begin{array}{c}\text { Left-Side } \\
\text { Saccade }\end{array}$} & & \multicolumn{2}{c}{$\begin{array}{c}\text { Right-Side } \\
\text { Saccade }\end{array}$} \\
Target Type & Location & $M$ & $S E M$ & & $M$ & $S E M$ \\
\hline Low frequency & Left & 6 & 4 & & 30 & 9 \\
& Right & 2 & 1 & & 30 & 6 \\
High frequency & Left & 1 & 1 & & 28 & 8 \\
& Right & 1 & 1 & & 30 & 6 \\
Functor word & Left & 4 & 2 & & 49 & 9 \\
& Right & 8 & 4 & & 49 & 8 \\
\hline
\end{tabular}


absence of spatially selective saccades toward the prior target location cannot be attributed to task demands, since the decision task in Experiment 2 explicitly demanded use of spatial knowledge.

Instead of using the target's spatial index to determine its prior sentence location, the readers may have scanned the representation of the sentence in verbal memory. If the target was reached early, after one or two words, a left decision could be made; it if was reached relatively late, a right decision could be made. This view can account for shorter decision times for left-side than for right-side targets, since they were encountered earlier in the memory scan. It can also account for the preponderance of right-directed saccades, which may have been external expressions of an analogous internal verbal memory scan that progressed from sentence onset to sentence offset.

In defense of spatial indexing, it could be argued that several procedural aspects of Experiments 1 and 2 hampered the execution of spatially selective saccades. First, the location of the probe word in the decision tasks, which was near the screen's center, differed from the location of the target, which was near the left or the right side of the screen. This mismatch could have interfered with the accessing of the previously read target's spatial index. Second, the decision task may have been probe centered in that the probe, rather than the target, was to be evaluated. This may have provided little incentive for movements of the eyes off the probe. Third, the previously read sentence was not visible during the memory task in the two experiments, which could have deprived the reader of an incentive to move the eyes to the spatial location of a previously read target. Regressions were less common and less accurate in Kennedy et al.'s (2003) experiments when the sentence with the regression target was no longer visible. Two additional experiments were conducted to examine whether readers would execute precise saccades to the location of a previously read target word under more natural reading conditions.

\section{EXPERIMENT 3}

In Experiment 3, spatial memory during silent sentence reading was examined in order to test whether readers access memory for the precise location of a previously read target word under more natural conditions. Sentence reading was no longer followed by a decision task, and the experimental task was thus no longer probe centered. Potential interference during the accessing of the target's spatial index was avoided by using targets and probes with a unique spatial location. An aim of Experiment 3 also was to determine whether an accuate regression to a previously read target is more likely when the word is visible in the periphery.

In addition, Experiment 3 removed the effects of a directional working memory scan on eye movements, which may have yielded a preponderance of right-directed saccades during the decision tasks in Experiments 1 and 2.
This was achieved by placing all the regression targets to the left of the starting point of a regression. Consequently, the direction of a saccade to a target word was now no longer analogous to the direction of a hypothesized verbal memory scan.

In Experiment 3, the participants read a one-line sequence of two sentences on each trial, which consisted of an initial fact-defining sentence that asserted a particular relationship (e.g., My mother is younger than my father) and a subsequent question that probed the comprehension of sentence content (e.g., Who was born earlier?). The second sentence contained a single word-referred to as the probe word - that referred to a specific word in the prior sentence (the target). In the two sample sentences, the probe and the target words were earlier and father, respectively. In contrast to Experiments 1 and 2, the probe and target words were never identical or associatively related. To find the answer to the question (the target), the meaning of the entire first sentence had to be established so that one of its words could be chosen as the answer.

In a sentence-removed condition, the sentence with the target word was no longer visible when the question with the probe word was read. This occurred on half of the trials. On the remaining trials, the initially read sentence and the question were visible throughout the experimental trial. If peripheral visibility of the regression target influences regression planning, regressions should be more common and more accurate when the sentence with the regression target remained visible in the periphery (Kennedy et al., 2003). Irrespective of the visibility of the target, readers should execute spatially selective regression to the target if the spatial index of a target word is retrieved during question answering.

\section{Method}

Participants. Thirty-six undergraduate students at the State University of New York at Binghamton participated for experimental course credit. None of them had participated in Experiment 1 or 2, and all gave informed consent before starting.

Apparatus. All the text was displayed on a light gray background on a 21-in. flat-screen Liyama Vision Master 510 monitor with $.28-\mathrm{mm}$ dot pitch that had a $1,028 \times 720$ pixel resolution. Black Courier type font was used so that each character occupied the same horizontal area of text, with a maximum of 12 horizontal pixels per character. In this experiment, head movements were not restrained, and reading distance was somewhat variable. At a typical distance of $85 \mathrm{~cm}$, each letter of text subtended approximately $0.44^{\circ}$ of visual angle laterally.

Eye movements were recorded via a head-mounted EyeLink pupil-tracking system. A high-speed video camera, used for the recording of pupil position, was positioned underneath the monitored eye and was held in place by a head-mounted gear. A second camera, mounted at the front of the headband, monitored head position. Both cameras sampled at a rate of $250 \mathrm{~Hz}$ throughout sentence reading, and the combined monitoring of pupil and head position yielded an absolute visual resolution better than $0.5^{\circ}$ (approximately the width of one letter) and a relative resolution of better than $0.25^{\circ}$.

Material and Design. Forty fact-defining sentences were generated that described common or fictional relationships. Each factdefining sentence contained between 5 and 10 words, 2 of which 
were candidate words, both of which were nouns. The two candidates were typically brought into a comparative relationship-for example, one of speed, age, or size. The first candidate was near the beginning of the first sentence, and the second candidate was at or near its end. For instance, in the sentence My mother is younger than my father, the 1st candidate word, mother, is spatially to the left of the second candidate, father, and the 2 candidate words are separated by 4 intervening words of various lengths. Each candidatecontaining sentence was followed by a question that contained a probe word. The probe word referred to one of the two candidates of the fact-defining sentence, denoting it as the target. The other candidate word is subsequently referred to as the distractor. Two questions were generated for each fact-defining sentence so that one candidate became the target of one question and the second candidate the target in the second question. The two questions were identical, except for the probe word that determined whether the first or the second candidate was the target-for example, Who was born earlier? and Who was born later?

The first (fact-defining) sentence and its question were constructed so that they occupied a single line of text (fewer than 80 characters) and so that the target was always to the left of the probe. Two lists were created so that the target-containing sentence was paired with a different question on each list. Half of the trials of each list contained a left-side target; the remaining trials contained a right-side target.

Two viewing conditions were created: one in which the factdefining sentence was visible throughout the experimental trial, and one in which it was removed from the screen when the reader moved the eyes from the fact-containing sentence onto the question. The two target conditions (left [far] vs. right [near] fact-defining sentence location) and reinspection conditions (visible vs. removed) are depicted in Figure 3.

Procedure. The experimental session began with a calibration of the eye-tracking system, during which the participants were asked to fixate a visual marker that appeared at three horizontal screen locations (left, center, and right) in random order. A calibration was considered accurate when the computed location of a fixation deviated by no more than one character space from the eyes' actual location.

Each trial began with a fixation cross appearing at the left side of the screen. Sentence onset was controlled by the subject by pressing the space bar on a computer keyboard. The participants began reading the two sentences on a line of text and were then asked to give the answer to the question by articulating the correct target. The reader's response was recorded by the experimenter and was used to determine accuracy rates. When unsure about the answer, the participants were told to guess. Both sentences were fully visible until the answer was provided on half of the trials. On the remaining trials, the fact-defining sentence was removed from the screen once the question had been read. An invisible boundary was defined, consisting of the blank space that separated the period of the first sentence from the first letter of the second sentence. Crossing of this boundary removed the first sentence in this condition and replaced it with a homogeneous light gray area. Another keypress terminated the visibility of the text, and yet another keypress started a new calibration of the system. The participants read 10 practice sentences and answered 10 practice questions prior to the presentation of the first experimental trial.

Measurement. As in Experiments 1 and 2, we analyzed gaze durations and skipping rates of candidate words as a function of their sentence location during sentence reading. Regressions leaving the question for the fact-defining sentence after probe word reading were of primary interest. For this, a criterion was established that defined a reinspection regression as a saccade that was launched from any one of the final 15 letter spaces of the question and arrived at one of the characters of the fact-defining sentence. Our working hypothesis was that these reinspection regressions would be directed at the target word because it was the answer to the question and because the target had to be articulated aloud before the next trial was initiated.

Four regression measures were computed as a function of target location: (1) regression rate (i.e., the relative frequency of regressions into the target-containing sentence), (2) regression size in letter spaces, (3) regression error, and (4) the landing position of the eyes at the regression target. Regression error consisted of the distance between the end point of a regression and the center of the target word, the goal point of spatially accurate short-range regressions (Radach \& McConkie, 1998). A regression that moved the eyes to the target's center thus yielded a zero-size regression error. Absolute error values were used to compute condition means, so that regression overshoot and undershoot would not cancel each other and yield pseudoaccurate movements. To determine landing position on the regression target, each target was partitioned into five equal-sized regions, and the frequency was recorded with which regressions reached one of the five segments. In case of a five-letter target, each letter constituted a different segment. All shorter and longer words were scaled. For instance, landings on Letters 1-3 of three-letter words were assigned to Segments 1, 3, and 5 , respectively.

\section{Results}

Sentence reading. As in Experiments 1 and 2, the location of the candidate word did not influence gaze durations (279 and $281 \mathrm{msec}$ for left and central positions, respectively; $F<1$ ), again indicating that there were no intrinsic differences between the two candidate locations in the fact-defining sentence. Skipping rates yielded a corresponding effect pattern, with similar skipping rates for left and central candidate locations [.2 and .22, respectively; $F(1,31)=1.15, p>.2]$.

Question answering. Question answering accuracy exceeded $96 \%$, indicating that both sentences were read for comprehension. Responses were slightly more accurate for far targets $(97.5 \%)$ than for near targets [95.4\%; $t(31)=2.37, p<.025]$. Trials in which the questions were answered inaccurately were discarded from the analyses of regressive eye movements.

Regressions. The results of the three regression indicators - regression rate, regression size, and regression error-are shown in Table 4 as a function of viewing condition and target position. Overall, the readers launched intersentence regressions from the question to the factcontaining sentence on $23.5 \%$ of the trials. Regression frequency did not vary as a function of target location $(F<1)$, but regressions occurred much more often when the first sentence remained visible for reinspection (43\%) than when it was erased upon question reading [4\%; $[F(1,31)=120.16, p<.001]$. The interaction between the target position and the reinspection visibility factors was negligible $[F(1,31)=1.53, p>.22]$.

Since there were relatively few intersentence regressions when the fact-containing sentence was no longer visible, only those conditions were subjected to statistical examinations of regression size and regression error in which the target-containing sentence was visible throughout a trial. As can be seen in Table 4, regressions in which the question was left for the target were 9 letter spaces larger for left-side targets than for right-side tar- 
Boundary locations, the two target words of the sentence, and the source word of the question are

typed in boldface:

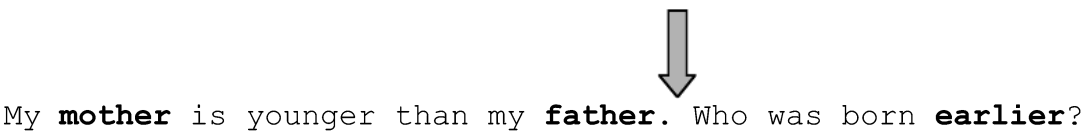

Sentence layout after boundary has been crossed (all four conditions are shown).

Sentence Present:

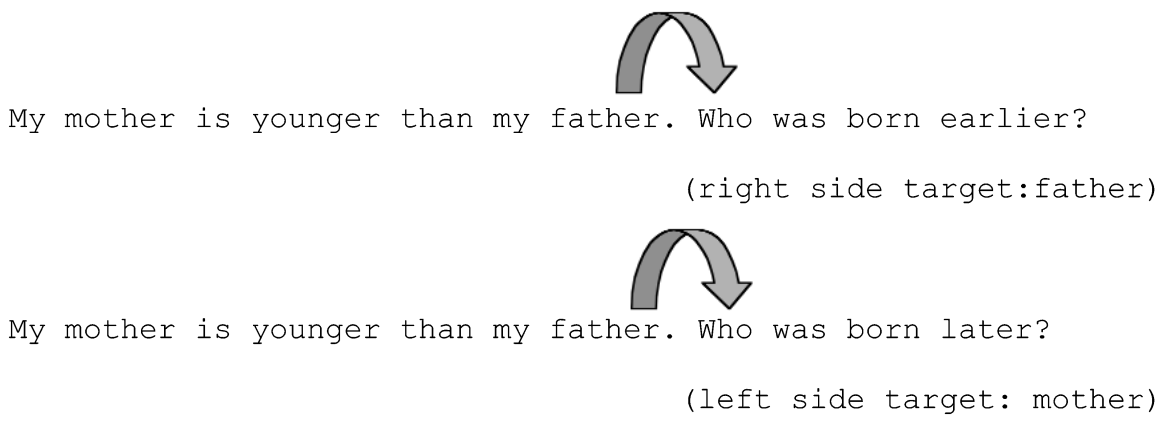

Sentence Absent:

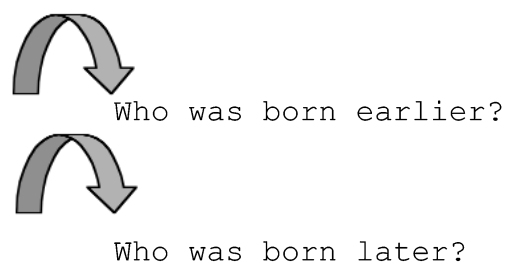

Figure 3. A sample trial in Experiment 3. The boundary location and the occurrence of the visual change are marked by arrows. Movement of the eyes to the right of the boundary resulted in the display change for the condition in which the sentence disappeared. For conditions in which the text remained visible, no changes occurred when the readers crossed the boundary.

gets $[t(31)=11.47, p<.01]$. Regressions rarely reached their hypothesized goal, however, the mean regression error being 17 letter spaces. Furthermore, regressions were less accurate when they were directed at a distant left-side target, with an error of 24 letter spaces, than when they were directed at a nearer right-side target, with an error of 10 letter spaces $[t(31)=8.15, p<.01]$.

The target was eventually fixated on virtually all of the trials in which a reinspection regression moved the eyes into the fact-defining sentence, however. An exam-

Table 4

Experiment 3: Relative Frequency (in Percentages) and Size and Error for Regressions (in Character Spaces) Launched From the 15-Character Probe Word Area When Targets Appeared in the First or Second Part of the Fact-Defining Sentence (With Standard Errors of the Means)

\begin{tabular}{|c|c|c|c|c|c|c|c|}
\hline \multirow[b]{3}{*}{ Condition } & \multirow{3}{*}{$\begin{array}{c}\text { Target } \\
\text { Location }\end{array}$} & \multicolumn{6}{|c|}{ Regression Measure } \\
\hline & & \multicolumn{2}{|c|}{ Rate } & \multicolumn{2}{|c|}{ Size } & \multicolumn{2}{|c|}{ Error } \\
\hline & & $M$ & $\overline{S E M}$ & $M$ & $\overline{S E M}$ & $M$ & $S E M$ \\
\hline \multirow[t]{2}{*}{ Target visible } & Left & 45 & 4 & 41 & 2.0 & 20.7 & 11.10 \\
\hline & Right & 41 & 4 & 32 & 1.5 & 11.7 & 6.62 \\
\hline \multirow[t]{2}{*}{ Target removed } & Left & 3 & 1 & 31 & & 37.0 & \\
\hline & Right & 5 & 1 & 17 & & 20.0 & \\
\hline
\end{tabular}


ination of the relative frequencies with which the five target landing positions were fixated revealed an even distribution, with relative frequencies of $17 \%, 18 \%$, $20 \%, 23 \%$, and $21 \%$ for Target Landing Positions $1-5$, respectively.

\section{Discussion}

Reinspection regressions into the target-containing sentence were relatively common in the sentence-visible condition. Moreover, these regressions were spatially selective as regression size increased with the distance of the target. Similar to the results in Kennedy et al. (2003), regressions were much less common and less accurate when the sentence with the target was erased from the screen prior to the regression.

Although these aspects of the data are in general agreement with the spatial-indexing hypothesis, there are other aspects to the data that are difficult to reconcile with it. Regression accuracy was higher for near than for far targets, which contradicts the assumption of equipotentiality, according to which each pertinent word in the previously read sentence should have been the recipient of a precise regression, irrespective of its sentence location. Moreover, intersentence regressions rarely reached the target, even when the distance to the target was relatively small, as occurred for right-side targets. The relatively large size of the regression error appears to contradict the explicit purpose of spatial indexing - to guide the eyes to the spatial location of a pertinent word with a high degree of spatial accuracy. Moreover, the distribution of landing positions of reinspection saccades that eventually placed the eyes onto the target was relatively flat, thus revealing no spatial selectivity. Shorter intraand interword regressions of up to 10 letter spaces, by contrast, direct the eyes at the word center (Radach \& McConkie, 1998). Precise spatial memory thus appears to guide regressions to locations that are very close to a fixation. Regressions to more distant targets appear to be guided by a different type of memory.

A small part of the regression error may be due to oculomotor factors, since the targeting error will increasingly undershoot with movement distance (Abrams \& Jonides, 1988). This account could explain undershoots of a few letter spaces. It cannot explain the much larger size of the regression error in Experiment 3. The source for this must be found elsewhere.

To some extent, the considerably lower spatial accuracy of reinspection saccades in Experiment 3 than in Kennedy et al.'s (2003) and in Kennedy and Murray's (1987) original experiments could be due to subtle differences in the materials. Although single-line presentations of sentences and probes (probes being words or short sentences) were used in all the studies, the probetarget relationship appears to have been more transparent in Kennedy and Murray's studies, where the target was identical to the probe word, synonomous to it, or the anaphoric referent of a subsequently encountered pronoun. The probe-target relationship in Experiment 3 was less transparent, since as the relationship between the two candidate words had to be examined after the question had been read. Under these conditions, the readers may not have immediately directed the eyes at the target. Instead, they may have fixated a segment of the factdefining sentence that assisted its comprehension, which could have been a word other than the target. Consequently, the regression error may have been relatively large, not because actual regressions were inaccurate, but because they were directed at words other than the target. In Experiment 4, this theoretical alternative was examined by using a novel experimental procedure that explicitly instructed the reader to regress to the target after question reading. According to the spatial-indexing hypothesis, reinspection regressions should now accurately move the eyes onto the target.

\section{EXPERIMENT 4}

Experiment 4 differed from Experiment 3 in that the identity of the regression target was experimentally controlled. This was achieved by telling the readers which word of the fact-defining sentence was the regression target. The regression target was spoken during question reading, rather than visually presented, so that its presentation would not establish a second spatial index for the target.

The same regression measures were computed as in Experiment 1, except for regression rates, since the execution of a regression to the target was now obligatory. Since the target of a regression was under explicit experimental control, we also computed two novel regression measures: the cumulated size of consecutive regressions toward the target and regression sequence error, which determined how much the cumulated sequence of regressions deviated from the target center.

\section{Method}

Participants. Thirty-two undergraduate students from the State University of New York at Binghamton participated for experimental course credit. None of them had participated in any of the previous experiments.

Apparatus, Materials, and Procedure. The apparatus, materials, and procedure were the same as those in Experiment 3, with one procedural exception. Specifically, a spatial boundary for the presentation of the spoken word was defined 15 characters to the left of the last character of the question. Upon crossing the boundary, the target of the regression was articulated over headphones, and the reader was instructed to move the eyes to the spatial location of the target in the fact-defining sentence, irrespective of whether it was still available for reinspection. The articulated target always provided the correct answer to the question. To encourage reading for meaning under these conditions, the participants were asked to repeat the fact-defining sentence and the question on approximately every 10 th trial.

Measurement. The same regression size measures were obtained as those in Experiment 1, with two important additions: the cumulated size of all consecutive regressions to the target and regression sequence error. The cumulated regression size consisted of all consecutive left-directed regressions toward the target until a counterdirectional right-directed eye-movement was executed. The 
regression sequence error consisted of the distance between the endpoint of a cumulated sequence of regressions and the center of the target. Since the regression target was presented over headphones during question reading, response accuracy could not be determined as was the case in Experiment 3. Instead, the readers were asked to report the fact-defining sentence after approximately every 10 th trial. Sentence report was virtually perfect, with an accuracy rate of $>95 \%$.

\section{Results}

Sentence reading. Examination of candidate word viewing duration during the reading of the fact-defining sentence showed once more no difference for left-side (far) versus right-side (near) candidate words [263 vs. 249 msec; $t(31)=1.18, p>.24]$, but candidates appearing to the left were now skipped less often than candidates occurring later in the fact-defining sentence [ $9 \%$ and $18 \%$, respectively; $t(31)=4.04, p<.01]$.

Regressions. Single-shoot regression size and singleshoot regression error are shown in Table 5 as a function of viewing condition and target location.

Similar to Experiment 3, single-shoot (standard) regressions to left-side targets were larger than regressions to right-side targets [42 and 31 letter spaces, respectively; $F(1,31)=44.99, p<.01]$. Single-shoot regressions were also larger when the fact-defining sentence remained available for reinspection than when it was removed [ 37 and 32 letter spaces, respectively; $F(1,31)=$ $9.18, p<.01]$. The two factors - target location and reinspection visibility-did not interact $(F<1)$.

Similar to single-shoot regressions, the cumulated regression size showed an effect of target location, with larger movements toward left side targets than toward right-side targets [49 and 36 letter spaces, respectively; $F(1,31)=110.79, p<.001]$. Target location and sentence visibility once more did not interact $[F(1,31)=$ $1.7, p>.20]$. In contrast to single-shoot regressions, however, cumulated consecutive regressions did not show an effect of sentence visibility $(F<1)$.

The single-shoot regression error in Experiment 4 was similar to the single-shoot regression error in Experiment 3. Once more, it was larger for left-side targets than for right-side targets [17 and 11 letter spaces, respec- tively; $F(1,31)=15.32, p<.01]$. Consistent with the results in Kennedy et al. (2003), the regression error also tended to be somewhat smaller when the sentence remained visible than when it disappeared (13 and 15 letter spaces, respectively), although this difference did not approach significance $[F(1,31)=2.51, p>.12]$. The interaction between the two factors was also not reliable $[F(1,31)=2.23, p>.14]$.

As can be seen in Table 6, the readers likely executed more than one consecutive regression toward the target in all the experimental conditions (all $t$ values $>8, p<.01$ ).

Similar to the single-shoot regression error, the resulting regression sequence error was slightly larger when the fact-defining sentence was removed from the screen $[F(1,31)=3.48, p<.08]$. Sentence visibility and target location did not interact $(F<1)$. The regression sequence error data revealed, however, a theoretically important deviation from the single-shoot regression error. Specifically, the effect of the target location was now reversed, with a smaller regression sequence error for left-side targets than for right-side targets [6 vs. 13 letter spaces, respectively; $F(1,31)=37.61, p<.01]$.

To determine whether explicitly demanded regressions to the target positioned the eyes near the target center, the distribution of landing positions on the target was calculated. Once more, only the sentence-visible condition was analyzed, since the regression sequence error was smaller in this condition. As in Experiment 3, the distribution was flat, with relative frequencies of $6 \%$, $32 \%, 20 \%, 19 \%$, and $22 \%$ for Positions $1-5$, respectively.

Comparison of single-shoot regressions in Experiments 3 and 4. Experiment 4 differed from Experiment 3 in that the readers were explicitly asked to execute a regression to the target on each trial. To examine whether the explicit demand to regress to the target influenced regression programming, single-shoot regressions in Experiment 4 were compared with single-shoot regressions in Experiment 3. Only the data from the sentence-present condition were used, since reinspection regressions were relatively rare in Experiment 3 when the fact-defining sentence was removed prior to the regression. The results were clear-cut: The readers launched larger regres-

Table 5

Experiment 4: Regression Size and Accuracy (With Standard Errors) as a Function of Target Location (Left [Far] or Right [Near] Located) and Sentence Visibility (Sentence Remains Visible or Is Removed)

\begin{tabular}{|c|c|c|c|c|c|c|c|c|c|}
\hline \multirow[b]{3}{*}{ Condition } & \multirow{3}{*}{$\begin{array}{l}\text { Target } \\
\text { Location }\end{array}$} & \multicolumn{4}{|c|}{$\begin{array}{c}\text { Standard } \\
\text { Regression Measure }\end{array}$} & \multicolumn{4}{|c|}{$\begin{array}{c}\text { Cumulative } \\
\text { Regression Measure }\end{array}$} \\
\hline & & \multicolumn{2}{|c|}{$\begin{array}{l}\text { Regression } \\
\text { Size }\end{array}$} & \multicolumn{2}{|c|}{$\begin{array}{l}\text { Regression } \\
\text { Error }\end{array}$} & \multicolumn{2}{|c|}{$\begin{array}{l}\text { Regression } \\
\text { Size }\end{array}$} & \multicolumn{2}{|c|}{$\begin{array}{l}\text { Regression } \\
\text { Error }\end{array}$} \\
\hline & & $M$ & SEM & $M$ & SEM & $M$ & $S E M$ & $M$ & $S E M$ \\
\hline \multirow{2}{*}{ Target visible } & Left side & 42 & 2 & 16 & 1 & 60 & 1 & 5 & 3 \\
\hline & Right side & 31 & 2 & 11 & 1 & 38 & 1 & 12 & 1 \\
\hline \multirow[t]{2}{*}{ Target removed } & Left side & 37 & 2 & 18 & 2 & 60 & 1 & 6 & 4 \\
\hline & Right side & 28 & 2 & 11 & 1 & 37 & 1 & 13 & 1 \\
\hline
\end{tabular}

Note-The table shows both the standard regression measure and the adjusted measure, which includes all consecutive regressions to the left. See the Results section for details. 
Table 6

Number of Consecutive Regressions in Experiment 4 Before the Target Word Was Reached

\begin{tabular}{cllll}
\hline & \multicolumn{4}{c}{ Target Location } \\
\cline { 2 - 5 } Condition & \multicolumn{2}{c}{ Left Side } & \multicolumn{2}{c}{ Right Side } \\
\cline { 2 - 5 } & $M$ & $S E$ & $M$ & $S E$ \\
\hline Target visible & 2.26 & 0.5 & 1.64 & 0.4 \\
Target removed & 2.24 & 0.4 & 1.76 & 0.4 \\
\hline
\end{tabular}

Note-Only those trials were analyzed in which the eyes were moved within five letter spaces of the target.

sions toward left-side targets, irrespective of experiment $[F(1,62)=54.68, p<.001]$. The absolute size of regressions toward left- and right-side targets was almost identical in the two experiments $(F<1)$, and the interaction of target location and experiment was negligible, $F<1$.

\section{Discussion}

Experiment 4 replicated the main findings of Experiment 3 , once more revealing spatially selective singleshoot regressions that were larger, although less accurate, when they were directed at a more distant left-side target than when they were directed toward a more proximal right-side target. Experiment 4 also showed that this occurred irrespective of the peripheral visibility of the regression target, although accuracy was slightly lower when the fact-defining sentence was no longer available for reinspection, as occurred in Kennedy et al. (2003).

The readers were explicitly instructed to move the eyes to the regression target in Experiment 4. They generally achieved this by executing a sequence of eye movements toward the target. A comparison of initial (singleshoot) regressions with subsequent regressions revealed notable differences. The initial single-shoot regression was relatively large; subsequent regressions were considerably smaller. Moreover, an examination of the fixation durations that preceded an initial and a consecutive regression showed large differences: The duration of the fixation that preceded the first regression was over $100 \mathrm{msec}$ longer $(348 \mathrm{msec})$ than the duration of the fixation that preceded the continuing regression $[236 \mathrm{msec}$; $t(31)=6.29, p<.01]$. This effect pattern suggests a division of labor: The initial regression generally moves the eyes in the vicinity of the target, and subsequent regressions home in on it.

Critically, Experiment 4 showed that this homing-in process was more successful for more distant left-side targets. Sensory and perceptual causes cannot be the source of this effect, since the left-side advantage occurred irrespective of the peripheral visibility of the target. Instead, a sequence of regressions moved the eyes closer to a left-side target because of a representational advantage. The potential nature of this advantage will be considered in the General Discussion section.

This interpretation of the results of Experiment 4 hinges on the assumption that the explicit instruction to regress did not lead to the adoption of task-specific regression strategies. Two findings support this view. First, factdefining sentences and their companion questions were read for meaning, since the participants accurately reported their content when asked. Critically, single-shoot regressions in Experiment 4 were virtually identical to naturally occurring single-shoot regressions in Experiment 3.

\section{GENERAL DISCUSSION}

The main goal of the present study was to test the spatial-indexing hypothesis, according to which readers store the spatial location of identified words during reading. Knowledge of spatial location is assumed to be subject to little deterioration and distortion, so that a word can be the target of a precise regression irrespective of its location when it becomes relevant during subsequent sentence or passage reading. The results of Experiments 1 and 2 were not in harmony with this assumption. In these experiments, the readers did not move the eyes back to the location of a previously read target word when it became relevant in a subsequent probe decision task.

Although regressions during sentence reading (Experiments 3 and 4 ) revealed some spatial selectivity, in that larger regressions were executed toward more distant regression targets, large reinspection (single-shoot) regressions rarely positioned the eyes at the target. Instead, sizable deviations from the target location were common, which violates the explicit purpose of spatial indexing, the reaching of a regression target via a single accurate regression. Notably, this lack of regression accuracy occurred irrespective of whether regressions occurred naturally in Experiment 3 or in response to explicit instruction in Experiment 4.

Instead of executing a single spatially accurate regression to a target word, the readers approached it via a sequence of regressions. In this approach, initial and subsequent regressions assumed functionally distinct roles. The initial regression tended to be relatively large, and it was preceded by a relatively long fixation duration. Its function appeared to be the positioning of the eyes in the vicinity of the target. The following regression(s) homed in on the target; they were smaller, and they were preceded by relatively short fixation durations. These small regressions were not spatially selective, however. As was noted before, small short-range regressions of up to 10 letter spaces move the eyes to the word center (Radach \& McConkie, 1998). In contrast to this, consecutive regressions in Experiments 3 and 4 did not direct the eyes to the center of the target.

Three of the present findings - the lack of spatial precision of single-shoot regressions, the execution of more than one regression to a target, and the effects of target location on regression error-are not in harmony with Kennedy and Murray's (1987; Kennedy et al., 2003) findings. One possible source of the discrepancy is the use of slightly different sentence materials. As was noted before, the sentence-probe relationship was less trans- 
parent in Experiments 3 and 4 than in Kennedy and Murray's studies. Another source is the use of different data selection criteria. The regression analyses in Experiments 3 and 4 included all regressions that moved the eyes from the last 15 letter spaces of the question into the fact-defining sentence. That is, we considered all regressions, irrespective of their size and where they positioned the eyes in the fact-defining sentence. Kennedy and Murray (1987; Kennedy et al., 2003) used a much more restrictive approach. In Kennedy et al., a regressive saccade was included in the analyses only when it was more than 16 letter spaces in size and only when it moved the eyes within 5 letter spaces of the designated target. These two criteria will necessarily increase regression accuracy and minimize the effects of target location, because inaccurate regressions are excluded from analyses.

Our results thus recommend a substantive modification of the spatial-indexing hypothesis, according to which memory for spatial location, like other types of memory, is relatively limited. Precise spatial knowledge appears to be available for relatively short durations, so that spatially precise regressions to the word center can be executed when the regression target is close to fixation (Hogaboam, 1983; Radach \& McConkie, 1998; Vitu \& McConkie, 2000). Regressions to more distant targets may not be guided by accurate spatial memory, however. This view is in harmony with other recent work on spatial memory, according to which memory for precise spatial location is subjected to relatively rapid deterioration (Fischer, 1999; Therriault \& Raney, 2002; Werner \& Diedrichsen, 2002), as little as $50 \mathrm{msec}$ in Werner and Diedrichson's study.

As the eyes move farther away from a word, precise spatial knowledge may give way to coarser coded spatial knowledge. Use of linguistic knowledge can thus supplement or replace use of spatial knowledge for regression planning. In either case, readers will be able to move the eyes in the general vicinity of the target, as did singleshoot regressions in Experiments 3 and 4. Critically, the precise target location appears to be searched for or inferred, a process that was overtly expressed in Experiment 4 by a sequence of consecutive regressions that homed in on the target.

In Experiment 4, the second stage of homing-in on the target was more accurate when it was a left-side targetthat is, when it was encountered early in the reading of the fact-defining sentence. Similar to this, Experiment 3 revealed a higher report accuracy rate for left-side targets, and Experiment 2 revealed a left-side advantage in the spatial decision task, with more accurate and faster decisions when the target had occupied a left-side location during prior sentence reading. Words that occurred early in a sentence may be the beneficiaries of primacy, as are words that occur early in a sequence of to-be-recalled words (e.g., Seiler \& Engelkamp, 2003). The accessibility of words in verbal memory, rather than the accessing of their spatial index, could thus determine the success with which readers home in on a distant regression target.

\section{REFERENCES}

Abrams, R. A., \& Jonides, J. (1988). Programming saccadic eye movements. Journal of Experimental Psychology: Human Perception \& Performance, 14, 428-443.

Baccino, T., \& Pynte, J. (1994). Spatial coding and discourse models during text reading. Language \& Cognitive Processes, 9, 143-155.

CARPENTER, P. A., \& DANEMAN, M. (1981). Lexical retrieval and error recovery in reading: A model based on eye fixations. Journal of Verbal Learning \& Verbal Behavior, 20, 137-160.

Christie, J. M., \& Just, M. A. (1976). Remembering the location and content of sentences in a prose passage. Journal of Educational Psychology, 68, 702-710.

EHRLICH, K., \& RAYNER, K. (1983). Pronoun assignment and semantic integration during reading: Eye movements and immediacy of processing. Journal of Verbal Learning \& Verbal Behavior, 22, 75-87.

FISCHER, M. H. (1999). Memory for word locations in reading. Memory, 7, 79-116.

Fischer, M. H. (2000). Perceiving spatial attributes of print. In A. Kennedy, R. Radach, \& J. Pynte (Eds.), Reading as a perceptual process (pp. 89-118). Amsterdam: Elsevier.

Frazier, L., \& RAYNER, K. (1982). Making and correcting errors during sentence comprehension: Eye movements in the analysis of structurally ambiguous sentences. Cognitive Psychology, 14, 178-210.

Greenberg, S. N., \& Koriat, A. (1991). The missing-letter effect for common function words depends on their linguistic function in the phrase. Journal of Experimental Psychology: Learning, Memory, \& Cognition, 17, 1051-1061.

HALL, D. C. (1974). Eye movements in scanning iconic imagery. Journal of Experimental Psychology, 103, 825-830.

Healy, A. (1994). Letter detection: A window to unitization and other cognitive processes in reading text. Psychonomic Bulletin \& Review, 3, 333-344.

Hogaboam, T. W. (1983). Reading patterns in eye movement data. In $\mathrm{K}$. Rayner (Ed.), Eye movements in reading: Perceptual and language processes (pp. 309-332). New York: Academic Press.

INHOFF, A. W., \& RADACH, R. (1998). Definition and computation of oculomotor measures in the study of cognitive processes. In G. Underwood (Ed.), Eye guidance in reading and scene perception (pp. 2954). Amsterdam: North-Holland.

INHOFF, A. W., \& RAYNeR, K. (1986). Parafovial word processing during eye fixations in reading: Effects of word frequency. Perception \& Psychophysics, 40, 431-439.

INHOFF, A. W., \& WegER, U. (2003). Advancing the methodological middle ground. In J. Hyönä, R. Radach, \& H. Deubel (Eds.), The mind's eye: Cognitive and applied aspects of eye movement research (pp. 335-344). Oxford: Elsevier.

KenNedy, A. (1987). Eye movements, reading skill and the spatial code. In J. R. Beech \& A. M. Colley (Eds.), Cognitive approaches to reading (pp. 169-186). Chichester, U.K.: Wiley.

KennedY, A. (1992). The spatial coding hypothesis. In K. Rayner (Ed.), Eye movements and visual cognition: Scene perception and reading (pp. 379-396). New York: Springer-Verlag.

KenNedy, A. (2000). Attention allocation in reading: Sequential or parallel? In A. Kennedy, R. Radach, D. Heller, \& J. Pynte (Eds.), Reading as a visual process (pp. 193-220). Oxford: Elsevier.

Kennedy, A., Brooks, R., Flynn, L.-A., \& Prophet, C. (2003). The reader's spatial code. In J. Hyönä, R. Radach, \& H. Deubel (Eds.), The mind's eye: Cognitive and applied aspects of eye movement research (pp. 193-212). Amsterdam: North-Holland.

KenNedy, A., \& MurRay, W. S. (1987). Spatial coordinates and reading: Comments on Monk (1985). Quarterly Journal of Experimental Psychology, 39A, 649-656.

Kolers, P. A. (1968). Foreword. In E. B. Huey, The psychology and pedagogy of reading (pp. xviii-xix). Boston: MIT Press.

KUČERA, H., \& FrANCIS, W. N. (1967). Computational analysis of presentday American English. Providence, RI: Brown University Press.

MalmberG, K. J., \& Nelson, T. (2003). The word frequency effect for recognition memory and the elevated-attention hypothesis. Memory \& Cognition, 31, 35-43. 
McConkie, G. W., Kerr, P. W., Reddix, M. D., \& Zola, D. (1988). Eye movement control during reading: I. The location of initial eye fixation on words. Vision Research, 28, 1107-1118.

RADACH, R., \& McConKIE, G. W. (1998). Determinants of fixation position in words during reading. In G. Underwood (Ed.), Eye guidance in reading and scene perception (pp. 77-100). Amsterdam: NorthHolland.

Rawson, K. A., \& Miyake, A. (2002). Does relocating information in text depend on verbal or visuospatial abilities? Psychonomic Bulletin \& Review, 9, 801-806.

RAYNER, K. (1979). Eye guidance in reading: Fixation locations within words. Perception, 8, 21-30.

RAYNER, K. (1998). Eye movements in reading and information processing: 20 years of research. Psychological Bulletin, 124, 372-422.

RAYNER, K., \& FrAZIER, L. (1987). Parsing teporarily ambiguous complements. Quarterly Journal of Experimental Psychology, 39A, 657673.

Rayner, K., \& Pollatsek, A. (1989). The psychology of reading. Englewood Cliffs, NJ: Prentice-Hall.

RichaRdSON, D. C., \& Spivey, M. J. (2000). Representation, space and Hollywood Squares: Looking at things that aren't there anymore. Cognition, 76, 269-295.

Seiler, K. H., \& Engelkamp, J. (2003). The role of item-specific information for the serial position curve in free recall. Journal of Experimental Psychology: Learning, Memory, \& Cognition, 29, 954-964.

Therriault, D. J., \& Raney, G. E. (2002). The representation and comprehension of place-on-page and text-sequence memory. Scientific Studies of Reading, 6, 117-134.

Vitu, F. M., \& McConkIE, G. W. (2000). Regressive saccades and word perception in adult perception. In A. Kennedy, R. Radach, \& J. Pynte (Eds.), Reading as a perceptual process (pp. 89-118). Amsterdam: Elsevier

Werner, S., \& Diedrichsen, J. (2002). The time course of spatial memory distortions. Memory \& Cognition, 30, 718-730.

Zechmeister, E. B., \& McKillip, J. (1972). Recall of places on the page. Journal of Educational Psychology, 63, 446-453.

Zechmeister, E. G., McKillip, J., Pasko, S., \& Bespalec, D. (1975). Visual memory for place on the page. Journal of General Psychology, 92, 43-52.

\section{NOTES}

1. One participant's manual decision times could not be determined because the keyboard's temporal interrupt was not recorded, due to hardware failure.

2 . No lateral saccades were recorded from 1 participant, due to equipment malfunction. Three participants' manual decision times could not be determined, because the keyboard's temporal interrupt was not recorded.

(Manuscript received April 7, 2003;

revision accepted for publication June 25, 2004.) 